\title{
A New Pathway for Incorporating the Impact of Solar Flares and Other Space Activity into Climate Modelling
}

\author{
Igor Suleimanov ${ }^{1}$, Sofya Prakhova ${ }^{2 *}$, Volker Rehbock ${ }^{2}$ \\ ${ }^{1}$ Department of Mathematics, Ufa State Petroleum Technological University, Ufa, Russia \\ ${ }^{2}$ Department of Mathematics and Statistics, Curtin University, Perth, Australia \\ Email: *sofya.prakhova@gmail.com
}

How to cite this paper: Suleimanov, I., Prakhova, S. and Rehbock, V. (2020) A New Pathway for Incorporating the Impact of Solar Flares and Other Space Activity into Climate Modelling. Journal of Applied Mathematics and Physics, 8, 2275-2285.

https://doi.org/10.4236/jamp.2020.810170

Received: March 5, 2020

Accepted: October 27, 2020

Published: October 30, 2020

Copyright (C) 2020 by author(s) and Scientific Research Publishing Inc. This work is licensed under the Creative Commons Attribution International License (CC BY 4.0).

http://creativecommons.org/licenses/by/4.0/

\begin{abstract}
A recent development in climate research is the recognition that space activity such as solar flares, gamma ray bursts, supernovas etc. can have a significant impact on the Earth. The existing methods of modelling space activity do not model these phenomena explicitly. Instead, their effect on ionization rates is formulated and then built into existing climate models. In this paper, based on the authors' earlier work, several analytical approaches for modelling a solar flare hitting the Earth are presented. These methods can also be used for modelling gamma-ray bursts or cosmic rays striking our planet. In addition, a method of calculating the total amount of radiation received during a solar flare is proposed. The future use of the proposed modelling approaches within comprehensive global climate models allows for explicit modelling of space activity and provides a new pathway for studying the possible effects of this activity on the Earth.
\end{abstract}

\section{Keywords}

Surface Integrals, Vector Field Theory, Solar Flares, Climate Implications

\section{Introduction}

During solar flares, highly energized protons can ionize air molecules, which then produce fast secondary electrons [1]. These electrons can then dissociate the nitrogen molecule, which results in the depletion of the total ozone column, acceleration of the zonal winds in polar jets, as well as in the cooling of surface air temperatures. This may even lead to extreme events. An example of such an event is the Carrington event in August-September 1859, which resulted in tele- 
graph failures all over Northern America and Europe and produced visible aurorae as far south as Cuba and Hawaii. Another example is a flare which occurred in March 1989, when power was knocked out across most of the Quebec province in Canada. Recently, much attention has focused on increasing our understanding of the Carrington event, in order to better quantify what extreme space events can do to our current technological society [2]. Events of this magnitude can also lead to satellite breakdown, with high associated financial costs. In addition, recent predictions indicate that such extreme events will be more likely over the coming decades [3].

There was a number of attempts to model the influence of large solar flares that took place in the past on the Earth [1] [2] [4] [5] [6] [7] [8]. In order to investigate their effect, certain increased ionization rates were assigned at different altitudes. For flares which occurred during the satellite era, the rates were calculated based on the proton flux or X-ray flux measurements (e.g. [4] [7]). In order to simulate solar flares which occurred before the spacecraft era, the rates of ionisation were either scaled up from the known events to an estimated intensity [1], or were taken from the ice core nitrate levels [2]. After assigning the ionisation rates the global climate model was run under specified conditions. The different chemical reactions which occur in the atmosphere and the transport processes are modelled.

Also, a number of papers attempted to model the effect of different forms of space activity, such as gamma-ray bursts [9] [10], cosmic rays [11] [12] [13] [14] and supernovas [15] [16] [17]. However, as for the modelling of solar flares, these activities were not modelled directly. Instead, their impact on various chemical species in the atmosphere was formulated and incorporated into global climate models.

In this paper, an overview of the approach for calculating insolation from the space perspective presented in the authors' work [18] is provided. The derivation of the model uses the methods of vector field theory and surface integrals. Next, three different approaches for explicit modelling of a solar flare striking the Earth are introduced. These approaches include modelling the solar flare as a vector, as well as modelling it as a cylinder with two different power distributions. These methods can also be used to model gamma-ray bursts or cosmic rays hitting the Earth. Finally, a formula for the total amount of radiation received during a solar flare event is derived.

\section{An Overview of the Approach for Calculating Insolation from the Space Perspective}

For an overall description, please see [18]. A summary of the approach along with a more detailed description of several aspects is given here.

Consider Figure 1.

In Figure 1, the radiation vector is

$$
\bar{a}(\alpha(t))=[F \sin (\alpha(t)),-F \cos (\alpha(t)), 0],
$$




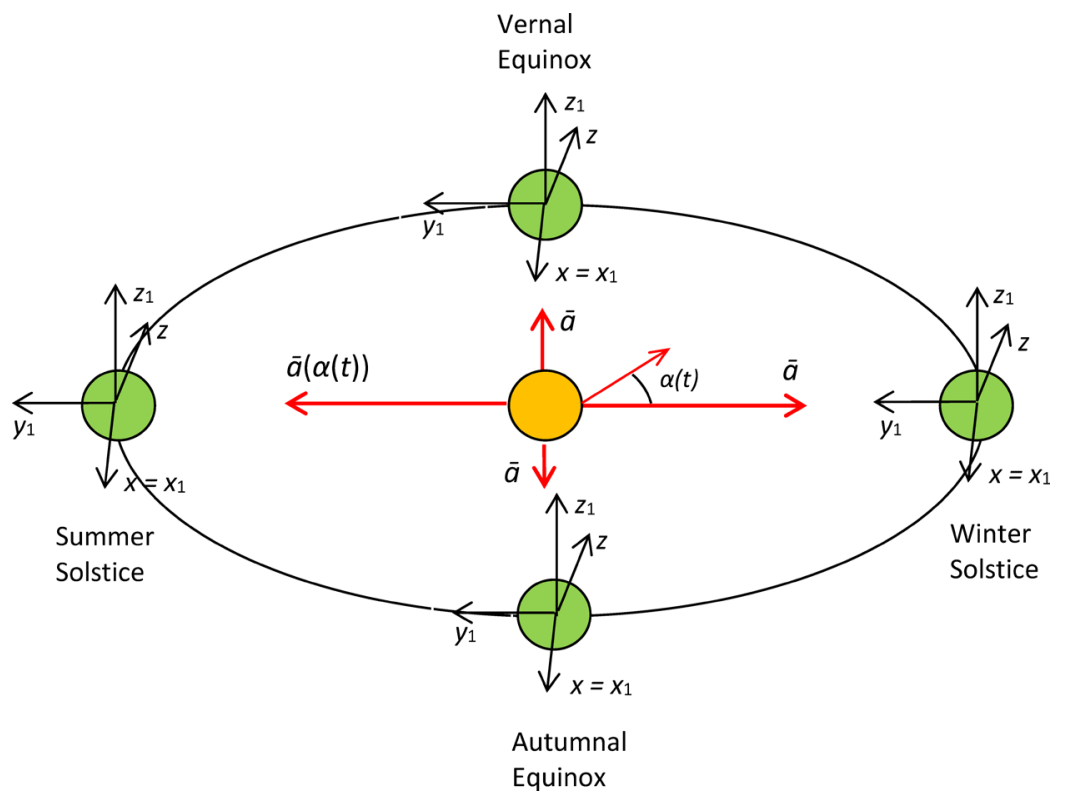

Figure 1. Coordinate systems and radiation vector (annual cycle). The vector $\bar{a}(\alpha(t))$ is the radiation vector, $\alpha(t)$ is the angle of the Earth's rotation around the Sun. The $(x, y, z)$ coordinate axes are aligned with the equatorial plane. The $\left(x_{1}, y_{1}, z_{1}\right)$ axes are aligned with the ecliptic plane.

where $F=1367 \mathrm{Wt} / \mathrm{m}^{2}$ is the solar constant. The angle of the Earth's rotation around the Sun $\alpha(t)$ ranges from $0^{\circ}$ to $360^{\circ}$, where $\alpha=0$ corresponds to the winter solstice. The Earth's orbit was assumed to be circular for the purpose of modelling. The $O Z$ axis represents the Earth's axis of rotation and $O Y$ is in the Earth's equatorial plane. The $\left(x_{1}, y_{1}, z_{1}\right)$ coordinate system is inclined to the equatorial plane by an obliquity angle which is $\varepsilon=23^{\circ} 26^{\prime}$ under present astronomical conditions. This coordinate system remains fixed as the Earth rotates around the Sun.

All the calculations were performed in the $\left(x_{1}, y_{1}, z_{1}\right)$ coordinates. The reason for this choice is that the formulation of the radiation vector remains the same as the Earth rotates around the Sun. The projection of the latitudinal belt in the $X_{1} O Z_{1}, X_{1} O Y_{1}$ and $Y_{1} O Z_{1}$ coordinate planes were obtained (see Figure 2). We should note that the Earth was assumed to be exactly spherical in our formulation. The projection obtained in the $Y_{1} O Z_{1}$ coordinate plane forms a nearly trapezoidal region, while the projections in the $X_{1} O Y_{1}$ and the $X_{1} O Z_{1}$ coordinate plane form ellipses. Since the projections obtained in the $Y_{1} O Z_{1}$ plane are the easiest for calculations, it was chosen for projecting a latitudinal belt in the $\left(x_{1}, y_{1}, z_{1}\right)$ coordinate frame.

The amount of light which is received by any particular area changes throughout the year. In the current approach it was modelled by an ellipse which changes with time since it is determined by the angle of the Earth's rotation around the Sun (see Figure 2). An ellipse change affects the change of size of the area receiving sunlight (the black hatched area in Figure 2) as well as size of the 
(a)

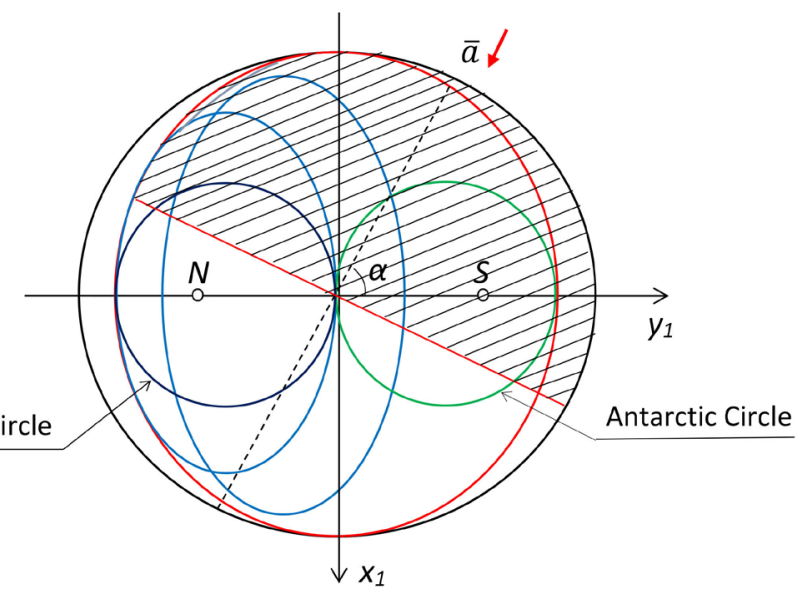

(b)

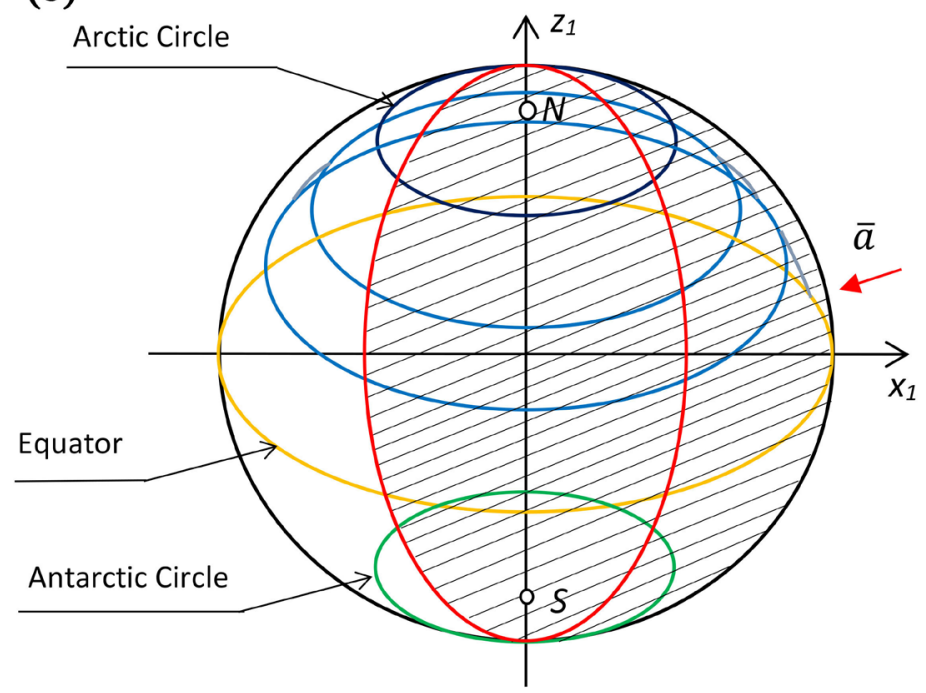

(c)

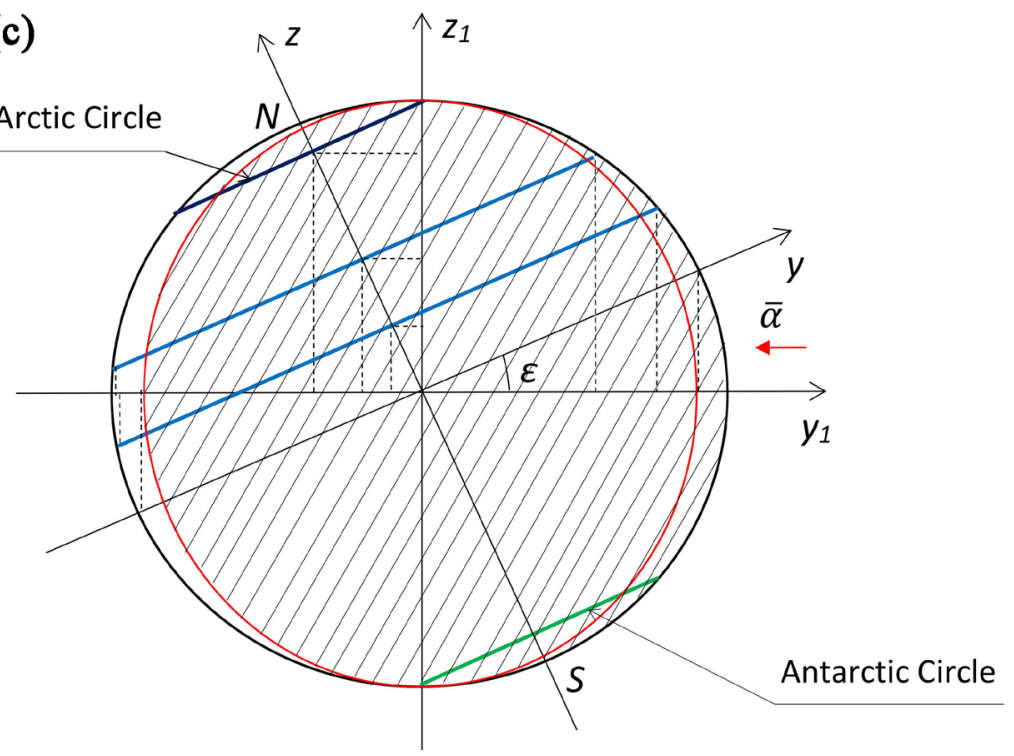

Figure 2. The projection of the latitudinal belt in the $X_{1} O Y_{1}(\mathrm{a}), X_{1} O Z_{1}$ (b) and $Y_{1} O Z_{1}$ (c) coordinate planes. The vector $\bar{a}$ is the radiation vector, $\varepsilon$ is the obliquity angle. The black hatched area is the area receiving sunlight. 
area of a latitudinal belt receiving sunlight (the illumination area). For different latitudes the illumination areas will be different. In addition, they may vary with time for some latitudinal belts. For details on this, please see [18].

The equations of the ellipse, circle, lower boundary of the belt and upper boundary of the belt were derived for the $Y_{1} O Z_{1}$ coordinate plane (see Figure 3).

In Figure 4, we show the direction of the radiation vector from the Earth's point of view and the surface orientation. The positively-oriented surface $\left(S^{+}\right)$in

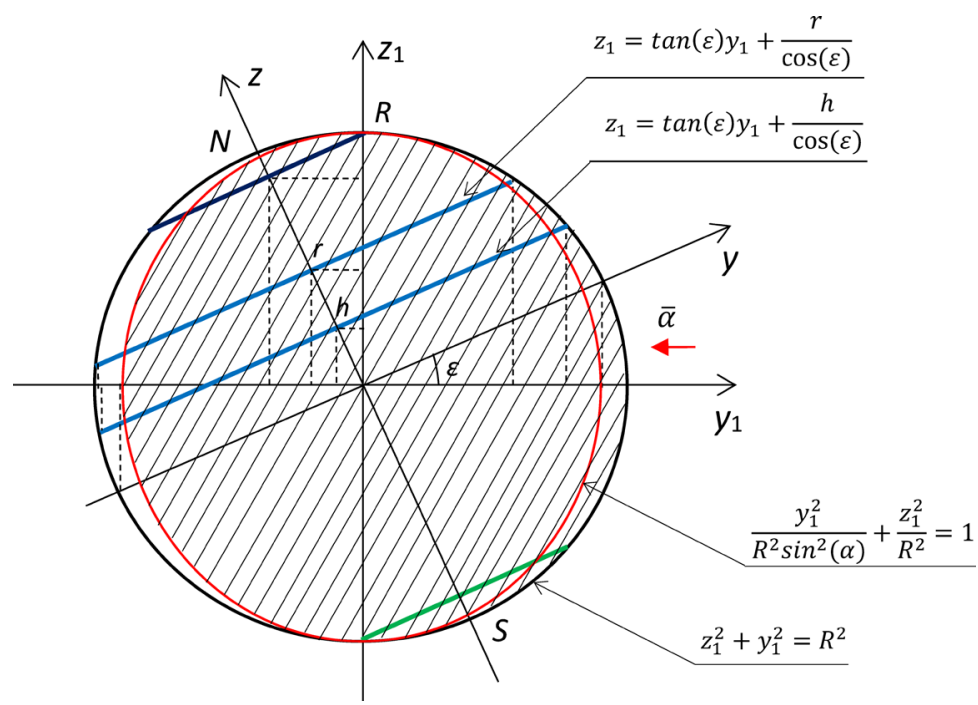

Figure 3. The equations of the ellipse, circle, lower boundary of the belt and upper boundary of the belt for the $Y_{1} O Z_{1}$ coordinate plane. The angle $\varepsilon$ is the obliquity angle, $\bar{a}$ is the radiation vector, $R$ is the Earth's radius, $h$ and $r$ are the perpendicular distances from the equatorial plane to the lower and upper latitude limits of the belt, respectively.

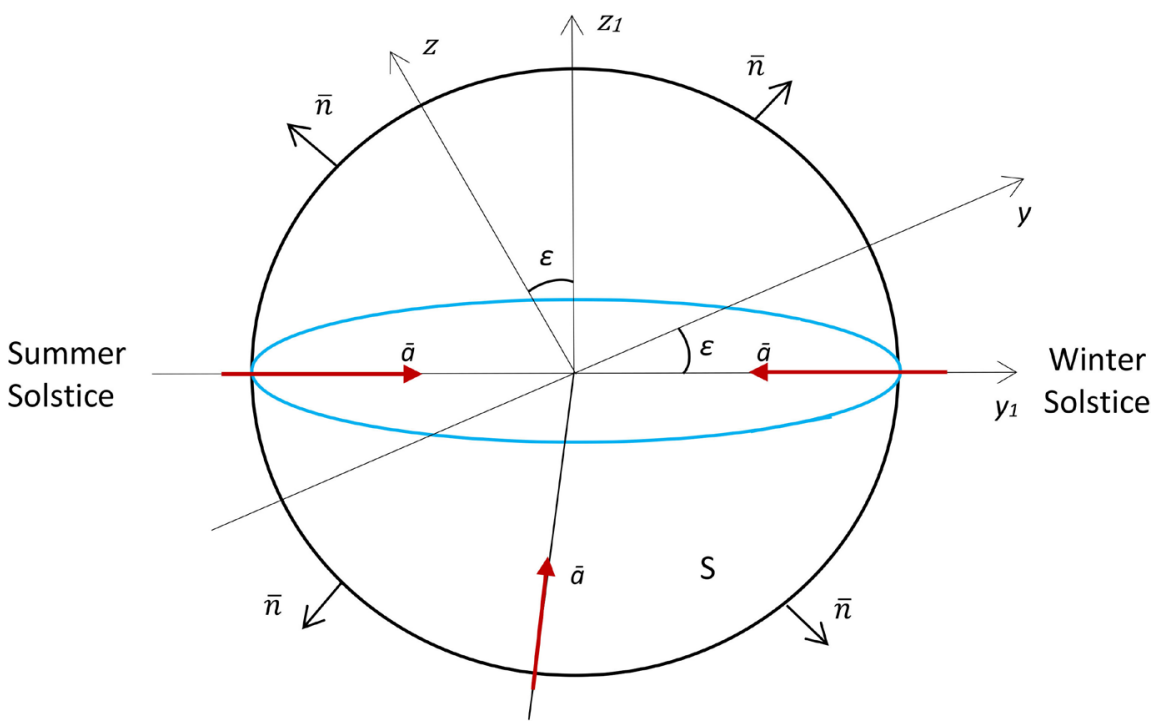

Autumnal

Equinox

Figure 4. The direction of the radiation vector from the Earth's point of view and the surface orientation. The vector $\bar{n}$ denotes the outward unit normal vector, $\bar{a}$ is the radiation vector and $\varepsilon$ is the obliquity angle. 
the chosen projection plane is the part of the sphere closer to the observer. The negatively-oriented surface $(S)$ is the part of the sphere pointing away from the observer.

The radiation flux through the surface per unit time (Flux) can be calculated as the product of the radiation vector $(\bar{a})$ and the outward unit normal vector $(\bar{n})$ to the surface $(S)$ integrated over the chosen side of the surface:

$$
\begin{aligned}
\text { Flux }= & \iint_{S}(\bar{a} \cdot \bar{n}) \mathrm{d} S \\
= & 2 F \sin (\alpha)-F \cos (\alpha) \int_{z_{1} \alpha^{+}}^{z_{1} \beta^{+}} \mathrm{d} z_{1} \int_{y_{1} \alpha^{+}}^{y_{1} \beta^{+}}\left(\frac{y_{1}}{\sqrt{R^{2}-y_{1}^{2}-z_{1}^{2}}}\right) \mathrm{d} y_{1} \\
& +F \cos (\alpha) \int_{z_{1} \alpha^{-}}^{z_{1} \beta^{-}} \mathrm{d} z_{1} \int_{y_{1} \alpha^{-}}^{y_{1} \beta^{-}}\left(\frac{y_{1}}{\sqrt{R^{2}-y_{1}^{2}-z_{1}^{2}}}\right) \mathrm{d} y_{1} .
\end{aligned}
$$

The lower $\left(z_{1} \alpha^{+}, y_{1} \alpha^{+}, z_{1} \alpha^{-}, y_{1} \alpha^{-}\right)$and upper $\left(z_{1} \beta^{+}, y_{1} \beta^{+}, z_{1} \beta^{-}, y_{1} \beta^{-}\right)$limits of integration were derived from the equations shown in Figure 3 separately for each type of illumination area.

The amount of radiation received per $\mathrm{m}^{2}$ in the current latitudinal belt (daily average) is the radiation flux through the surface divided by the surface area of the latitudinal belt $\left(S_{\text {belt }}\right)$ :

$$
I=\frac{\text { Flux }}{S_{\text {belt }}} .
$$

The surface area of latitudinal belt was computed by the equation below.

$$
S_{\text {belt }}=2 \pi R(r-h) \text {. }
$$

For the derivation details, please see [18].

Based on the approach outlined above, the amount of insolation for a complete year cycle for any latitudinal belt was computed in Maple software with the time step of approximately 10 days. We have also calculated the annual average value for each latitudinal belt and compared it with satellite data from NASA (see [18]). The results show very good agreement for equatorial and middle latitudes region with an average accuracy of $98 \%$. Slightly less agreement (94.5\%) can be observed in the polar regions which are known to be difficult to model. The average accuracy of the approach is $97 \%$.

\section{The Mathematical Formulation of Solar Flares and Other Space Activity}

In this section, building on the work introduced in Section 1, several different ways of modelling solar flares are presented. The methods are introduced in their order of complexity in the first three subsections. Finally, a method of calculating the total amount of radiation received in a solar flare event is proposed.

Different types of space activity may also be included into the modeling in a similar way, i.e. gamma-ray bursts or cosmic rays. 


\subsection{Modelling a Solar Flare as a Vector}

Firstly, we aim to model a solar flare simply as an additional radiation vector coming from the Sun and entering the Earth at an angle $\phi$. This, together with solar radiation vector, is illustrated in Figure 5.

The flare radiation vector $\bar{a}_{f}$ was assigned as:

$$
\bar{a}_{f}=\left[-N_{f} \cos (\phi),-N_{f} \sin (\phi), 0\right],
$$

where $N_{f}$ is the power of flare.

A flare radiation flux per unit time can be calculated as:

$$
\text { Flux }_{\text {flare }}=\iint_{S}\left(\bar{a}_{f} \times \bar{n}\right) \mathrm{d} S,
$$

where $S$ is the surface area of the Earth and $\bar{n}$ is the outward unit normal vector.

This formulation of the flare radiation flux is similar to the computation of the regular radiation flux. Different types of radiation may also be included into the modelling in a similar way, i.e. gamma-ray bursts or cosmic rays.

\subsection{Modelling a Solar Flare as a Cylinder with Power Distribution Depending on Magnitude of Position Vector}

Next, we propose to model radiation flux released as the result of a solar flare via a cylinder with a certain radius $R_{a}$ (see Figure 6). However, the power of the flare

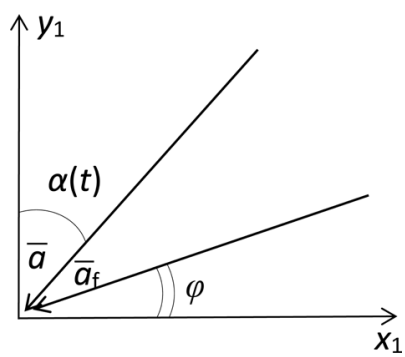

Figure 5. The radiation vector $\bar{a}$ and flare radiation vector $\bar{a}_{f}$ coming from the Sun. The angle $\alpha(t)$ is the angle of the Earth's rotation around the Sun, $\phi$ is the angle at which a flare enters the Earth.

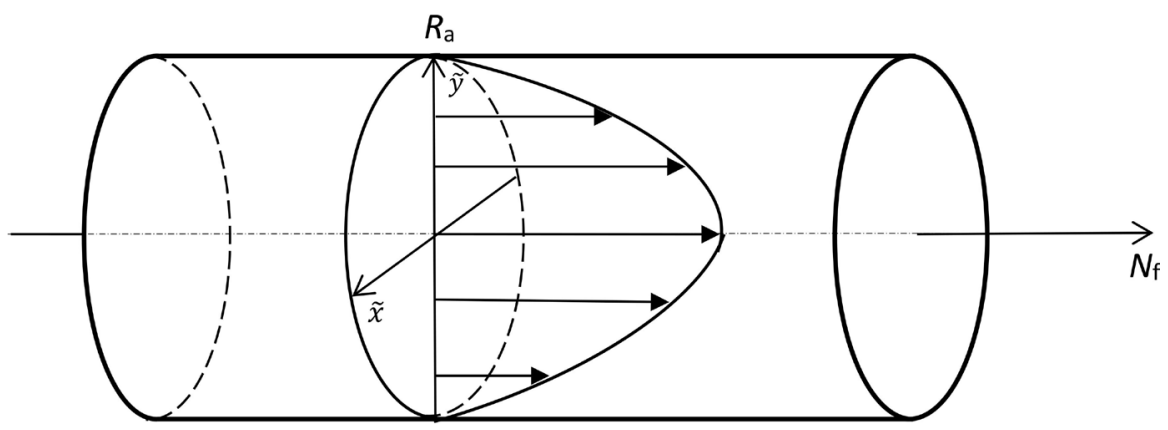

Figure 6. Approximate distribution of flare power inside the cylinder. The $\tilde{x}$ and $\tilde{y}$ axes form an intermediate coordinate system. The radius $R_{a}$ is the radius of the cylinder, $N_{f}$ is the power of flare. 


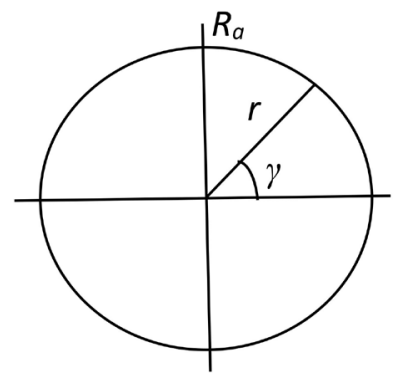

Figure 7. Polar coordinate system for assigning a flare power at any point of the cylinder. The radius $R_{a}$ is the radius of the cylinder.

is different in different areas of the cylinder. In particular, it is the highest in the centre and decreases towards the edges of the cylinder. This is illustrated by arrows of a different length in Figure 6.

In order to assign a flare power at any point of the cylinder, a polar coordinate system is introduced (see Figure 7).

Since flare power depends on radial distance and does not depend on polar angle, power at any point on a cross section of the cylinder can be defined as:

$$
\frac{N_{f}}{2}\left(\cos \left(\frac{\pi r}{R_{a}}\right)+1\right)
$$

In order to include the whole area of the cross section, the following integral was calculated:

$$
\int_{0}^{2 \pi} \mathrm{d} \gamma=2 \pi
$$

Therefore, flare power distribution within a cylinder can be assigned as:

$$
f(r)= \begin{cases}\frac{N_{f}}{2} 2 \pi\left(\cos \left(\frac{\pi r}{R_{a}}\right)+1\right), & \text { if } r \in\left[0, R_{a}\right], \\ 0, & \text { if } r \notin\left[0, R_{a}\right] .\end{cases}
$$

The flare radiation vector then can be written as:

$$
\bar{a}_{f}=[-f(r) \cos (\phi),-f(r) \sin (\phi), 0] .
$$

This modelling approach allows us to take into account the variation of flare power within a flare flux.

\subsection{Modelling a Solar Flare as a Cylinder with Power \\ Distribution Depending on Magnitude of \\ Position Vector and Time}

In addition to the uneven distribution of flare power within a flux, it also varies as flux passes the Earth. This can be incorporated into the modelling by assigning a flare power with a certain distribution. From the literature [19] [20], as well as guidance by a diagram from the NASA website ${ }^{1}$, a gamma distribution was chosen to describe this effect:

${ }^{1}$ http://hesperia.gsfc.nasa.gov/hessi/images/diagrams.gif. 


$$
N_{f}(t)= \begin{cases}N_{f} \frac{t-t_{1}}{\Gamma(2)} \exp \left(-\left(t-t_{1}\right)\right), & \text { if } t \in\left[t_{1}, t_{2}\right], \\ 0, & \text { if } t \notin\left[t_{1}, t_{2}\right],\end{cases}
$$

where $t_{1}$ corresponds to the start of the flare and $t_{2}$ corresponds to the end.

The distribution of flare power within a cylinder and the flare radiation vector then take the form:

$$
f(r, t)= \begin{cases}\frac{N_{f}(t)}{2} 2 \pi\left(\cos \left(\frac{\pi r}{R_{a}}\right)+1\right), & \text { if } r \in\left[0, R_{a}\right], \\ 0, & \text { if } r \notin\left[0, R_{a}\right],\end{cases}
$$

and

$$
\bar{a}_{f}=[-f(r, t) \cos (\phi),-f(r, t) \sin (\phi), 0],
$$

respectively.

The proposed way of modelling solar flares allows for the incorporation of radiation dispersal at the beginning and end of a flare flux due to entropy.

\subsection{Calculation of Total Amount of Radiation during Solar Flare}

The total amount of radiation received per $\mathrm{m}^{2}$ in the Earth is the sum of the amount of regular solar radiation and the amount of radiation received from a flare:

$$
I_{\text {total }}=I_{\text {main }}+I_{\text {flare }} \text {. }
$$

The amount of regular solar radiation during a flare can be calculated as:

$$
I_{\text {main }}=\frac{\int_{t_{1}}^{t_{2}} \iint_{S}(\bar{a} \times \bar{n}) \mathrm{d} S \mathrm{~d} t}{S} .
$$

The amount of radiation received from a flare can be found as:

$$
I_{\text {flare }}=\frac{\int_{t_{1}}^{t_{2}} \int_{0}^{R_{a}} \iint_{S}\left(\bar{a}_{f} \times \bar{n}\right) r \mathrm{~d} S \mathrm{~d} r \mathrm{~d} t}{S},
$$

where $r$ is the Jacobian determinant.

\section{Conclusions and Future Work}

In this paper, built on the authors' earlier work, a number of different approaches for explicit modelling of a solar flare striking the Earth are presented. These methods allow for taking into account the variation of flare power within a flare flux, as well as for the incorporation of radiation dispersal at the beginning and end of a flare flux due to entropy. In addition, it can be possible to model gamma-ray bursts or cosmic rays hitting the Earth in a similar way. Finally, the cumulative radiation received in a solar flare event is determined.

We should note that it was assumed that the flare flux is wide and covers the Earth completely. The cases when the flux is narrow and covers only a certain 
area of the Earth have not been considered so far. However, the most complex of the proposed approaches allows for modelling these cases in the future. In order to do this, the intersection of the surface area of a cylinder and the surface area of the Earth has to be calculated.

The future use of the proposed modelling approaches within global climate models will enable to explicitly model space activity within the models and to study the possible impact of this activity on our planet.

\section{Conflicts of Interest}

The authors declare no conflicts of interest regarding the publication of this paper.

\section{References}

[1] Calisto, M., Verronen, P.T., Rozanov, E. and Peter, T. (2012) Influence of a Carrington Like Event on the Atmospheric Chemistry, Temperature and Dynamics. Atmospheric Chemistry and Physics, 12, 8679-8686. https://doi.org/10.5194/acp-12-8679-2012

[2] Rodger, C.J., Verronen, P.T., Clilverd, M.A. and Seppälä, A., Turunen, E. (2008) Atmospheric Impact of the Carrington Event Solar Protons. Journal of Geophysical Research: Atmospheres, 113, D23302. https://doi.org/10.1029/2008JD010702

[3] Barnard, L., Lockwood, M., Hapgood, M., Owens, M.J., Davis, C.J. and Steinhilber, F. (2011) Predicting Space Climate Change. Geophysical Research Letters, 38, L16103. https://doi.org/10.1029/2011GL048489

[4] Qian, L., Wang, W., Burns, A.G., Chamberlin, P.C., Coster, A., Zhang, S.R., et al. (2019) Solar Flare and Geomagnetic Storm Effects on the Thermosphere and Ionosphere during 6-11 September 2017. Journal of Geophysical Research: Space Physics, 124, 2298-2311. https://doi.org/10.1029/2018JA026175

[5] Jackman, C.H., Cerniglia, M.C., Nielsen, J.E., Allen, D.J., Zawodny, J.M., McPeters, R.D., et al. (1995) Two-Dimensional and Three-Dimensional Model Simulations, Measurements, and Interpretation of the Influence of the October 1989 Solar Proton Events on the Middle Atmosphere. Journal of Geophysical Research: Atmospheres, 100, 11641-11660. https://doi.org/10.1029/95JD00369

[6] Thomas, B.C., Jackman, C.H. and Melott, A.L. (2007) Modeling Atmospheric Effects of the September 1859 Solar Flare. Geophysical Research Letters, 34, L06810. https://doi.org/10.1029/2006GL029174

[7] Verronen, P.T., Seppälä, A., Clilverd, M.A., Rodger, C.J., Kyrölä, E., Enell, C.F., et al. (2005) Diurnal Variation of Ozone Depletion during the October-November 2003 Solar Proton Events. Journal of Geophysical Research: Space Physics, 110, A09S32. https://doi.org/10.1029/2004JA010932

[8] Krivolutsky, A., Vyushkova, T.Y. and Mironova, I. (2017) Changes in the Chemical Composition of the Atmosphere in the Polar Regions of the Earth after Solar Proton Flares (3d Modeling). Geomagnetism and Aeronomy, 57, 156-176. https://doi.org/10.1134/S0016793217020074

[9] Thomas, B.C., Melott, A.L., Jackman, C.H., Laird, C.M., Medvedev, M.V., Stolarski, R.S., et al. (2005) Gamma-Ray Bursts and the Earth: Exploration of Atmospheric, Biological, Climatic, and Biogeochemical Effects. The Astrophysical Journal, 634, 509. https://doi.org/10.1086/496914 
[10] Ejzak, L.M., Melott, A.L., Medvedev, M.V. and Thomas, B.C. (2007) Terrestrial Consequences of Spectral and Temporal Variability in Ionizing Photon Events. The Astrophysical Journal, 654, 373-384. https://doi.org/10.1086/509106

[11] Carslaw, K., Harrison, R. and Kirkby, J. (2002) Cosmic Rays, Clouds, and Climate. Science, 298, 1732-1737. https://doi.org/10.1126/science.1076964

[12] Usoskin, I.G. and Kovaltsov, G.A. (2006) Cosmic Ray Induced Ionization in the Atmosphere: Full Modeling and Practical Applications. Journal of Geophysical Research: Atmospheres, 111, D21206. https://doi.org/10.1029/2006JD007150

[13] Usoskin, I., Gladysheva, O. and Kovaltsov, G. (2004) Cosmic Ray-Induced Ionization in the Atmosphere: Spatial and temporal Changes. Journal of Atmospheric and Solar-Terrestrial Physics, 66, 1791-1796. https://doi.org/10.1016/j.jastp.2004.07.037

[14] Svensmark, J., Enghoff, M., Shaviv, N. and Svensmark, H. (2016) The Response of Clouds and Aerosols to Cosmic Ray Decreases. Journal of Geophysical Research: Space Physics, 121, 8152-8181. https://doi.org/10.1002/2016JA022689

[15] Thomas, B.C., Melott, A.L., Fields, B.D. and Anthony-Twarog, B.J. (2008) Superluminous Supernovae: No Threat from $\eta$ Carinae. Astrobiology, 8, 9-16.

https://doi.org/10.1089/ast.2007.0181

[16] Melott, A., Thomas, B.C., Kachelriess, M., Semikoz, D. and Overholt, A. (2017) A Supernova at 50 pc: Effects on the Earth's Atmosphere and Biota. The Astrophysical Journal, 840, 105. https://doi.org/10.3847/1538-4357/aa6c57

[17] Thomas, B.C., Engler, E., Kachelrieß, M., Melott, A., Overholt, A. and Semikoz, D. (2016) Terrestrial Effects of Nearby Supernovae in the Early Pleistocene. The Astrophysical Journal Letters, 826, L3. https://doi.org/10.3847/2041-8205/826/1/L3

[18] Prakhova, S., Suleimanov, I. and Rehbock, V. (2014) A New Approach for Modelling Insolation from the Space Perspective. Applied Mathematical Sciences, 8, 2951-2964. https://doi.org/10.12988/ams.2014.43204

[19] Charakhch'yan, A., Tulinov, V. and Charakhch'yan, T. (1962) Energy Spectrum and Time Dependence of the Intensity of Solar Cosmic-Ray Protons. Soviet Physics Jetp, 14, 530-538.

[20] Meyer, P., Parker, E. and Simpson, J. (1956) Solar Cosmic Rays of February, 1956 and Their Propagation through Interplanetary Space. Physical Review, 104, 768-783. https://doi.org/10.1103/PhysRev.104.768 